\title{
GESTIÓN DEL CONOCIMIENTO: UNA ESTRATEGIA INNOVADORA PARA EL DESARROLLO DE LAS UNIVERSIDADES
}

\section{KNOWLEDGE MANAGEMENT: AN INNOVATIVE STRATEGY FOR THE DEVELOPMENT OF UNIVERSITIES}

\author{
Jaime Alberto Beltrán-Ríos ${ }^{1}{ }^{\mathbb{D}}$, José Antonio López-Giraldo ${ }^{\circledR}$, Claudia Gelvez-Velásquez ${ }^{3}$, Shirley Quintero- \\ Benítez $^{4}$ y Viviana Katerine Benítez-Cardona ${ }^{5}$
}

\author{
${ }^{1}$ Universidad Católica Luis Amigó, Colombia.Email: jaime.beltranri@amigo.edu.co \\ ${ }^{2}$ Universidad Católica Luis Amigó, Colombia. Email: jose.lopezgi@amigo.edu.co \\ ${ }^{3}$ Universidad Católica Luis Amigó, Colombia. Email: clagev1974@gmail.com \\ ${ }^{4}$ Universidad Católica Luis Amigó, Colombia. Email: shirlyqb@gmail.com \\ ${ }^{5}$ Universidad Católica Luis Amigó, Colombia. Email: vivi.benitez1130@gmail.com
}

Para citar este artículo: Beltrán, R. J., López, G. J., Gelvez, V. C., Quintero, B. S. y Benítez, C. V. (2019). Gestión del conocimiento: una estrategia innovadora para el desarrollo de las universidades. Clío América, 13(26), 362-369. doi: http://dx.doi.org/10.21676/23897848.3513

Recibido: 27 junio de 2019

Aceptado: 03 de octubre de 2019

Publicado en línea: noviembre 28 de 2019

Palabras clave:

gestión del conocimiento; estrategia; innovación; universidades.

JEL: 032 .

\section{RESUMEN}

La actual sociedad del conocimiento responde a las crecientes demandas del entorno, producto de los cambios constantes generados por el progreso del conjunto de técnicas en la información y la comunicación, la globalización y la apertura de mercados, y gestiona el conocimiento como estrategia para el incremento de capacidades individuales y el desarrollo de las universidades en el mundo de hoy. Este activo intangible gestionado eficientemente fortalece los procesos de innovación en los distintos sectores académicos donde se implemente, fomentando, incluso, el desarrollo de innovaciones con impacto social de cara al desarrollo de los países. Para una adecuada gestión del conocimiento se deben alinear las estrategias y las políticas de las instituciones de educación superior con este proceso, además de comprender los elementos, las herramientas y los modelos que intervienen, para lograr los resultados esperados con la implementación del modelo en cuanto a la generación de valor, el desarrollo de ventajas institucionales y la permanencia en el mercado a través de la competitividad. En consecuencia, este artículo dispone de una reflexión basada en artículos de investigaciones que resaltan la importancia de la gestión del conocimiento, orientada al desarrollo y la innovación en las instituciones de educación superior.

\section{ABSTRACT}

The current knowledge society responds to the growing demands of the environment, as a result of the constant changes generated by the progress of a set of techniques in information and communication, globalization and markets opening, managing knowledge as a strategy for an increase of individual capacities, and the development of universities in the today's world. This intangible asset, when efficiently managed, strengthens the innovation processes in the different academic sectors where it is implemented. This promotes the development of innovations with social impact, facing the countries' development. For adequate knowledge management, the strategies and policies of the organization must be aligned with this process, in addition to understanding the elements, tools, and models involved, achieving the expected results with the implementation of the model in terms of value generation, institutional advantages development and permanence in the market through business competitiveness. Consequently, this article has a reflection based on research articles that highlight the importance of knowledge management, oriented towards development and innovation in higher education institutions. 


\section{INTRODUCCIÓN}

En un entorno globalizado, caracterizado por cambios continuos, desarrollos tecnológicos, creciente competencia en el mercado y cambios en las necesidades de los clientes, las organizaciones deben responder a las demandas de manera innovadora y competitiva a través de la generación de valor y el desarrollo de capacidades corporativas. Por eso, la Gestión de Información y la Gestión del Conocimiento contribuyen a la toma de decisiones estratégicas, pues a partir de la información y el conocimiento se pueden generar y determinar mejores alternativas de decisión y la mejor solución ante una situación-problema (Rodríguez, 2015), y fomentar el desarrollo de capacidades tanto individuales como organizacionales (Olsson y Acevedo, 2015), entre otras ventajas que otorgan valor a las empresas basadas en el conocimiento.

Pese a la creciente implementación de la gestión del conocimiento en las instituciones de educación superior, y demás estamentos sociales y económicos, los resultados en cuanto a innovación de nuevos productos, servicios y procesos, o la generación de nuevos conocimientos no se logra, dado que la gestión implica una serie de elementos, herramientas y modelos que no son llevados a cabo con eficiencia para que la gestión del conocimiento sea eficaz y logre generar ventajas competitivas sostenibles.

Este estudio persigue la comprensión de las actuales sociedades y economías del conocimiento que reconocen en este activo intangible un recurso que revoluciona las estrategias de competitividad empresarial, orientadas a la innovación organizacional y con impacto social a través de la gestión eficiente del conocimiento.

\section{METODOLOGÍA}

Para la elaboración de este documento se realizó una búsqueda y lectura de diferentes artículos en bases electrónicas y revistas en línea. Fue de tipo documental, con la idea de identificar algunos elementos importantes que orientaran acciones de innovación y desarrollo en los centros de educación superior, y reflexionar sobre los elementos innovadores que proporciona la gestión del conocimiento a las instituciones universitarias.

El enfoque es cualitativo, pues se realiza una revisión bibliográfica de tipo descriptivo con fuentes por categoría.

\section{Fundamentación teórica:}

\section{Gestión del conocimiento}

Si bien el conocimiento ha sido tema de interés filosófico desde los griegos, analizando la relación entre el individuo que busca el conocimiento y la 'cosa' que se conoce o se busca conocer (Ortega, Hernández y Tobón, 2015), en el campo organizacional ha cobrado vital importancia desde 1970 con Peter Drucker, quien acuñó el término Knowledge worker, concepto que ha evolucionado de manera sorprendente hasta lo que conocemos hoy como la gestión del conocimiento organizacional (Correa, Benjumea y Valencia, 2019). Por su lado, las universidades tienen unos objetivos misionales, además de un compromiso social, que puede apalancarse desde la gerencia del conocimiento que circula dentro de las instituciones para producir nuevos conocimientos, innovar o transferir los ya generados. Este proceso requiere comprender el qué, quién, cómo y para qué gestionar la información y los conocimientos en la organización.

\section{El conocimiento}

Los conocimientos se clasifican en conocimiento tácito y conocimiento explícito (Pérez, Solano y Amezcua, 2019), y comprenden el 'qué' de la gestión del conocimiento, que implica la búsqueda de su origen, bien en las personas o en los documentos, libros, revistas científicas, archivos organizacionales, entre otros. Cuando se habla de know how se hace alusión a un conocimiento que es propio de las personas, de la experiencia que ha adquirido a partir de la utilización del conocimiento a su quehacer diario; es un conocimiento que actúa, al aplicarse de manera inconsciente (Pérez, Solano y Amezcua, 2019).

Existen diferencias entre datos, información y conocimiento (López y Beltrán, 2018). Los datos son cifras vacías en sí mismas. La información permite que estos datos, a la luz de un contexto específico, puedan decir algo de la realidad que se describe; pero el conocimiento organizacional es la capacidad orgánica para generar nuevos conocimientos, diseminarlos entre los miembros de una organización y materializarlos en productos, servicios y sistemas (Nonaka, como se citó en López y Beltrán, 2018). Por lo anterior, es necesario comprender la diferencia entre estos conceptos, dado que, si bien las empresas tienen un sistema de información, no significa que el almacenamiento de esta información en una organización esté estructurado (Torres y Lamenta, 2015), o genere conocimientos a nivel individual y colectivo, agregando valor a la compañía.

\section{Las personas y la organización:}

En el 'quién' de la gestión del conocimiento se habla de los 
agentes a nivel personal o individual, grupal o colectivo, y organizacional. Son los agentes donde se puede "depositar" el conocimiento para que se puedan gestionar y generar los resultados esperados, es decir, tal como lo expresa Nonaka y Takeuchi (como se citó en López y Beltrán, 2018), el conocimiento se puede expandir en una espiral de conocimiento que va desde el conocimiento tácito, propio de los individuos, hacia el conocimiento explícito que puede ser utilizado por los grupos y las organizaciones, a partir de un proceso de socialización, externalización, combinación e internalización del conocimiento. El conocimiento es un proceso de 'complementariedad' donde cada participante desde su diversidad, sus capacidades y sus talentos aporta, recibe y transforma conocimientos (Betancourt y Giraldo, 2018).

\section{El proceso}

De acuerdo con Valero, López y Pirela (2017), en la gestión del conocimiento se ponen en juego procesos, procedimientos, medios, herramientas y planes que se articulan para llevar a cabo una gestión efectiva que permita crear, organizar, transferir y aplicar conocimientos. En este sentido, el 'cómo' de la gestión del conocimiento hace referencia a unos elementos necesarios que se conjugan y se dinamizan con base en unos modelos; así, cada organización adapta o adopta modelos ya existentes, o crea su propio modelo, que establece los procesos, los medios y las herramientas con las cuales se gestiona el conocimiento. Dentro de estos elementos encontramos herramientas de base tecnológica como los portales web (Valero et al., 2017), la cultura, la comunicación corporativa y el recurso tecnológico (Christer y Acevedo, 2015): también personas, procesos y tecnologías (Medina, Nogueira, Medina, Medina y Assafiri, (2018), y el clima organizacional (Torres, Lamenta y Hamidian, 2018).

Existen modelos que buscan evaluar la madurez en el proceso de gestión del conocimiento. Siguiendo a Montañez y Lis (2017), los modelos de madurez son guías para la ejecución de la gestión del conocimiento que brindan a las organizaciones la posibilidad de valorar sus iniciativas en diferentes esferas, al indicar un continuo que va del estado presente de la organización y describe las vías para dirigirse hacia el estado ideal de la gestión del conocimiento. Sin embargo, de acuerdo con lo formulado por Torres y Lamenta (2015), el diseño y la ejecución de un modelo de gestión del conocimiento no es suficiente per se para el alcance de los resultados esperados, pues es indispensable alinear este modelo de gestión con los planes estratégicos de la organización y comprometer a todas las personas que la integran para que se construyan ambientes de socialización, donde el aprendizaje y el intercambio de conocimientos sea accesible, dinamizado y fortalecido por cada uno de los colaboradores.

\section{RESULTADOS}

Christer y Acevedo (2015) encuentran una correlación entre la gestión del conocimiento y el desarrollo de capacidades profesionales en el profesorado de instituciones de educación superior, a partir de la generación de espacios de comunicación e intercambio de conocimientos entre los equipos de trabajo. Por su parte, Mirabal (2015) plantea que la gestión del conocimiento contribuye a forjar organizaciones inteligentes, con capacidades para resolver problemas, adaptarse a los cambios de manera estratégica y eficaz, fomentando y manteniendo la competitividad en el mercado. Se reconoce el conocimiento como el factor generador de riqueza y de desarrollo a través del fortalecimiento de la capacidad de aprendizaje de las organizaciones y de los individuos (Díaz, Valdez y Quintana, 2018). En las instituciones de educación superior, Correa, Benjumea y Valencia (2019) expresan que la gestión del conocimiento contribuye a la mejora en la toma de decisiones trascendentales, especialmente en temas de malla curricular y procesos educativos, y además de genera valor y fortalece la innovación organizacional

Los aportes de la gestión del conocimiento organizacional también son a nivel generacional (Buitrago, Serna y Rodríguez, 2018), en tanto que la documentación de los procesos posibilita la utilización de las lecciones aprendidas como información útil para la toma de decisiones acertadas, y una gestión empresarial efectiva que dirija a las empresas hacia la competitividad a través de la generación de valor.

\section{Gestión del conocimiento e innovación}

La innovación como forma de desarrollo se hace importante para las instituciones educativas del siglo XXI, pues proporciona valor agregado y múltiples beneficios para ellas. Las características de la gestión del conocimiento que más impactan en la educación superior son la investigación y la innovación; ambas son pilares determinantes para el desarrollo de las universidades. La principal característica de la innovación es que emplea muy variadas formas de utilización del conocimiento. Por lo anterior, para que se impriman procesos innovadores en el sector educativo, así como en las empresas del sector real, el colaborador debe conocer sus fortalezas y avanzar en ellas, debe recibir retroalimentación, afianzar sus propias experiencias $\mathrm{y}$, a su vez, fortalecer las áreas que le competen con el fin de no perder tiempo en actividades que 
no son de su alcance. Suleman (2006) muestra los cuatro factores que influyen básicamente en el flujo del conocimiento dentro de la organización empresarial, los cuales se encuentran distribuidos de la siguiente manera: canales de comunicación, actitud individual, actitud de grupo y cultura de la organización. Todos los anteriores son los mismos factores que determinan el éxito en la gestión del conocimiento en las universidades.

Canales de comunicación: Se refiere al aprovechamiento del lenguaje utilizado como herramienta de comunicación plasmada en libros, documentos técnicos, bases de datos, portales de internet, seminarios y comunidades de práctica.

Actitud individual: Esta actitud se ve representada en el ser como elemento dinamizador de la creación y la transferencia de conocimientos de otros individuos en un equipo y, en segunda instancia, las actitudes tales como el respeto mutuo y la igualdad.

Actitud de grupo: Se basa en la idea de que los miembros de las estructuras orgánicas, por percepción natural, propenden a conformar grupos donde se presenta el intercambio de conocimientos, la sinergia y la empatía.

Cultura de la organización: Describe fundamentalmente a la cultura organizacional, la cual se desarrolla a través de los mitos, las leyendas y las políticas empresariales.

En la búsqueda de mayores estándares de calidad, las IES (Instituciones de Educación Superior) se han volcado a la tarea de mejorar sus procesos, cualificar permanentemente su personal, innovar, investigar y producir conocimiento básico y aplicado que permita mejorar las condiciones de vida de toda la sociedad (Henao, López y Garcés; 2014). Una de las herramientas importantes en la innovación universitaria es el libro digital, pues este permite utilizar la tecnología como herramienta de interacción al servicio de la academia y de las necesidades de los estudiantes, que ven en la tecnología un elemento de transformación que les posibilita mayor conocimiento, interactividad, nuevos formatos, distribución de la información a menor costo y exploración de nuevas herramientas.

\section{Instituciones de educación superior}

Las sociedades actuales experimentan unos procesos acelerados en términos de expansión y creatividad, lo que ha llevado a ciertos niveles de crisis en aspectos económicos, sociales, políticos, religiosos y otros. Esto consecuentemente las hace parecer desactualizadas, ya que parecieran no acercarse a los propósitos para los que fueron creadas, es decir se ven disminuidas en sus competencias. Ante este panorama, las organizaciones y los centros de educación superior se ven comprometidos, ya que pareciera que están en desventaja para atender las demandas sociales, administrativas, organizacionales y/o académicas del medio. Sin duda eso es lo que motiva a todas las instancias de educación superior y otras a desarrollar acciones y/o estrategias que les permitan avanzar en sus conocimientos, intentando desde ahí generar valor a cada una, creando factores diferenciadores en términos de productividad, y motivando el aumento de capital intelectual y competitividad. Tales procesos permiten que las universidades inviertan en aspectos tan vitales como la investigación, tanto cualitativa como cuantitativa, pues ambas generan valor en tanto permiten crear otros programas académicos, servicios para las comunidades educativas y otros tantos para la sociedad en general. Sin embargo, habría que mencionar que tales procesos de investigación en ocasiones se ven entorpecidos con aspectos relacionados con lo presupuestal y con la baja disponibilidad de recursos tecnológicos e informáticos. Eso dificulta dar comienzo a nuevas ideas de innovación y desarrollo, además de la inexistencia de planes estratégicos institucionales que puedan avalar la propuesta de investigación y las propuestas organizacionales que planteen los beneficios y/o el alcance real para las universidades, en términos de sostenibilidad, desarrollo y tecnología. En algunos casos se vislumbra una resistencia al cambio por parte de aquellos que administran las instituciones, a la par de la inexistencia de equipos de formación en investigación y desarrollo sostenible. Estos aspectos, en conjunto o de manera individual, obstaculizan el proceso de crear conocimiento en y desde las Instituciones de Educación Superior.

Para desarrollar adecuados procesos de formación y de difusión del conocimiento, es determinante el material de apoyo. Es así que "juega un papel importante el material de apoyo a la docencia en proceso educativo que es soporte básico de la gestión documental, y es esta información la que requiere ser gestionada desde el conocimiento" (Ramos, 2015). Por demás, esto

implica generar y difundir una estrategia de gestión del conocimiento que articule acciones fundamentales como entender necesidades y oportunidades del conocimiento, construir conocimientos relevantes para el negocio, organizar y distribuir el conocimiento de la organización, crear condiciones para la aplicación del conocimiento de la organización y explotar el conocimiento. (Ramos, 2015)

No hay que olvidar que 
Las universidades son actores clave en el tejido social por su desempeño en actividades de formación y docencia, investigación y vinculación con el entorno socio-económico. Estas tres misiones han estado presentes desde el origen mismo de las universidades, aunque la presencia relativa de dichas misiones haya variado a lo largo del tiempo y según el tipo de universidades. (Quevedo, Morquecho, Vásquez y Neira 2019)

Así, teniendo en cuenta lo que va exigiendo la globalización, además de cada uno de los lineamientos de los entes que regulan conjuntamente con la constitución, las IES han empezado a pensarse y ampliar las relaciones con las sociedades para participar activamente en su desarrollo económico.

\section{DISCUSIÓN}

Mediante la gestión del conocimiento, cuando se establecen los ambientes reales o virtuales propicios, las organizaciones favorecen que el individuo se desarrolle en su trabajo y aporte ideas. Así, se evita la fuga de conocimiento que se da cuando las personas abandonan la organización, (Arias, Cruz, Pedraza, Ordoñez y Herrera, 2007). Al respecto, Nuñez et al. (como se citó en Yong, Rodríguez y Ruso, 2017) plantea que la innovación se produce a partir de la aplicación del conocimiento ya adquirido en nuevos y variados problemas, de la transformación de este conocimiento previo para crear nuevos conocimientos, y de la acomodación de estos conocimientos previos con otros nuevos para solucionar problemas, crear nuevos productos o mejorar los procesos. Estos procesos de I+D que cumplen objetivos particularmente empresariales, pueden ser trascendidos desde la universidad para complementar este I+D con la responsabilidad de las IES de desarrollar conocimiento con impacto social.

Desde este punto de vista, las organizaciones aprovechan el conocimiento y las innovaciones producto de su gestión, implementando el enfoque de I+D para agregar valor a sus procesos y productos. Mientras tanto, las universidades y las IES orientan el proceso de innovación hacia intereses sociales, además de los productivos, para responder o dar solución a las problemáticas de orden social de la comunidad donde está inmersa.

Pese a las diferencias en los objetivos que persiguen organizaciones productivas e Instituciones de Educación Superior, el interés por la generación y la aplicación de conocimientos para adaptarse a los cambios incesantes es mutuo. Es relevante reconocer la trascendencia de las alianzas entre empresa y universidad, por su impacto en cuanto a la creación y la aplicación de nuevos conocimientos. Las universidades concebidas como las organizaciones clave en la sociedad del conocimiento, cuya razón de ser se centra en la creación de conocimiento e investigación, dan apertura a los procesos de innovación que los demás sectores de la economía pueden aprovechar para mejorar su competitividad, agregar valor a sus productos y procesos, y adaptarse a los cambios del entorno.

La alianza interinstitucional constituye entonces un factor que aporta significativamente a la transferencia, al desarrollo y a la aplicación de conocimientos con impacto en los resultados y en la competitividad organizacional. Desde este punto de vista, Buitrago, Serna y Rodríguez (2018) señalan que la documentación de las experiencias y los conocimientos producto de estas experiencias, contribuyen al aprendizaje generacional y permiten que este conocimiento quede en las organizaciones y no solo en las personas. Las sociedades del conocimiento reconocen en este activo intangible el recurso estratégico para responder a las exigencias de la globalización y a los constantes desarrollos tecnológicos, no solo para adaptarse de manera eficiente a estos cambios, sino también para ser generadores de cambio y mantenerse en el mercado a través de la innovación y la generación de ventajas competitivas sostenibles.

\section{CONCLUSION}

La gestión del conocimiento es un tema muy en boga en la actualidad y ha cobrado gran importancia en las IES, dado que el conocimiento es su principal herramienta. Por ello, se ha encontrado que los procesos que gestionan el conocimiento desde las universidades deben generar el impacto social y de innovación que permita aplicar estos conocimientos a la solución de los problemas de la comunidad, cumpliendo así con sus objetivos misionales.

Es fundamental que las personas que integran las universidades se comprometan con el desarrollo y la generación de capacidades. Esto solo es posible desde la gerencia, fortaleciendo una cultura del aprendizaje, del reconocimiento del conocimiento como motor de desarrollo, no solo desde su producción, sino desde su aplicación para la innovación y para la solución de problemas colectivos.

Las organizaciones que gestionan el conocimiento deben enfocar sus esfuerzos en el 'quién' del proceso, y no solo en el 'cómo'. Es decir, deben otorgar especial valor a las 
personas como elemento fundamental para la generación de conocimientos y capacidades organizacionales, y no solo a las herramientas o procesos.

Administrar la información no es gestionar el conocimiento; tener una cultura de aprendizaje no implica transferir o generar saberes; implementar un modelo para gestionar el conocimiento no es suficiente para desarrollar, socializar y crear sapiencias. Es indispensable interrelacionar todos los componentes de la gestión del conocimiento para obtener los resultados esperados en la generación de valor: las personas, las políticas y los planes organizacionales, la tecnología, la cultura y la comunicación organizacional, entendiendo que cada uno de estos, como componentes de un proceso sistémico, son interdependientes, y el cambio en alguna de las partes afecta inherentemente a las otras partes y al todo.

Entonces, la responsabilidad y el compromiso que tienen todas las IES derivan de un proceso de resignificación, que implica también desaprender las antiguas formas de orientar lo administrativo, lo organizacional y lo funcional. Sin duda, este nuevo orden permite una nueva cultura institucional que esté orientada por los objetivos, además de que favorezca a toda la sociedad y la comunidad educativa en todas las necesidades a intervenir.

\section{Declaración sobre conflictos de interés}

Los autores manifestamos que somos independientes con respecto a las instituciones financiadoras y de apoyo, y manifestamos que durante la ejecución del trabajo y la redacción del manuscrito no han incidido intereses o valores distintos a los que usualmente tiene la investigación.

\section{REFERENCIAS BIBLIOGRÁFICAS}

Arias, J., Cruz, H., Pedraza, M., Ordoñez, A. y Herrera, L. (2007). Los escenarios de la gestión del conocimiento y el capital intelectual en los procesos de investigación. Signo y Pensamiento, $X X V I$ (50),

81.

https://www.redalyc.org/pdf/860/86005

006.pdf

Betancourt, M. y Giraldo, J. (2018). La gestión del conocimiento desde la perspectiva del pensamiento del mestizaje. Revista Interamericana de Bibliotecología, 41(2), 205-213. 10.17533/udea.rib.v41n2a08

Buitrago, A., Serna, H. y Rodríguez, M. (2018). La gestión del conocimiento empresarial como contribución al aprendizaje generacional-estudio de caso en Colombia. Horizontes Empresariales, 17(1), 45-58.

http://revistas.ubiobio.cl/index.php/HHE E/article/view/3281

Correa, A., Benjumea, M. y Valencia, A. (2019). La gestión del conocimiento: Una alternativa para la solución de problemas educacionales. Revista Electrónica Educare, 23(2), 1-27. http://dx.doi.org/10.15359/ree.23-2.1.

Christer, J. y Acevedo, J. (2015) El desarrollo de capacidades y la gestión del conocimiento en los potenciales de cambio y competitividad universitaria. Revista Anagramas, 14(27), 201-216-. http://www.scielo.org.co/scielo.php?pid $=\mathrm{S} 1692-$

$\underline{5222015000200011 \& \text { script }=\text { sci_abstract }}$ $\underline{\text { \&tlng }=\mathrm{es}}$

Díaz, G., Valdez, M. y Quintana, M. (2018). La gestión del conocimiento en el ámbito empresarial. UNIANDES EPISTEME: Revista de Ciencia, Tecnología e Innovación, $\quad 5(2), \quad$ 133-148. http://45.238.216.13/ojs/index.php/EPISTE 
$\underline{\text { ME/article/view/1015 }}$

Henao, E., López, M. y Garcés, R.

(2014). Medición de capacidades en

investigación e innovación en

instituciones de educación superior: una

mirada desde el enfoque de las

capacidades dinámicas.

Entramado,10(1).

253.

https://www.redalyc.org/pdf/2654/2654

31574016.pdf

López, J. y Beltrán, J. (2018).

Fundamentación de un modelo de gestión del conocimiento para la

Universidad Católica Luis Amigó. Medellín, Colombia: Fondo Editorial Universidad Católica Luis Amigó. https://www.funlam.edu.co/uploads/fon doeditorial/434_Fundamentacion_de_un _modelo_de_gestion.pdf

Medina, D., Nogueira, D., Medina, A.,

Medina, Y. y Assafiri, Y. (2018). Modelo conceptual para la gestión del conocimiento mediante el observatorio. Ingeniería Industrial, 39(3), 283-290. https://www.redalyc.org/articulo.oa?id= $\underline{3604 / 360458817007}$

Mirabal, J. (2015). Gestión dinámica de conocimiento organizacional. Enlace: Revista Venezolana de Información, Tecnología y Conocimiento, 12(2), 5578.

https://www.redalyc.org/pdf/823/82340995 005.pdf

Montañez, L. y Lis, J. (2017). A propósito de $\underline{\text { los modelos de madurez de gestión del }}$ conocimiento. rev.fac.cienc.econ, $\underline{X X V(2),}$ 65.

https://www.redalyc.org/pdf/909/90952 679005.pdf

Olsson, J. y Acevedo, J. (2015). El desarrollo de capacidades y la gestión del conocimiento en los potenciales de cambio y competitividad universitaria. Anagramas Universidad de Medellín, 14 (27), 203. http://www.scielo.org.co/pdf/angr/v14n 27/v14n27a11.pdf

Ortega, M., Hernández, J. y Tobón, S. (2015). Análisis documental de la gestión del conocimiento mediante la cartografía conceptual. Revista $R a$ Ximhai, 11(4), 141-160. https://www.redalyc.org/articulo.oa?id= 461/46142596009

Pérez, N., Solano, C. y Amezcua, M. (2019) Conocimiento tácito: características en la práctica enfermera. Gaceta Sanitaria, $33(2)$, 191-196. https://doi.org/10.1016/j.gaceta.2017.11. $\underline{002}$

Quevedo, J., Morquecho, J., Vásquez, L. y Neira, M. (2019). Manual didáctico de emprendimiento para la incubadora de empresas dirigido a estudiantes 
GESTIÓN DEL CONOCIMIENTO: UNA ESTRATEGIA INNOVADORA PARA EL DESARROLLO DE LAS UNIVERSIDADES

universitarios. Revista Arbitrada

Interdisciplinaria KOINONIA, IV(8),

663.

file:///C:/Users/pc/Downloads/Manual_dida

ctico de emprendimiento para la incubad.

pdf

Ramos, A. (2015). Gestión del

conocimiento en el proceso de docencia

para instituciones de educación

superior. SIGNOS, 7(2), 31-43.

file:///C:/Users/pc/Downloads/Dialnet-

GestionDelConocimientoEnElProcesoDeDo

cenciaParaIns-6726211.pdf

https://www.redalyc.org/articulo.oa?id=

$5604 / 560458751003$

Rodríguez, Y. (2015). Gestión de Información y del Conocimiento para la toma de decisiones organizacionales. Bibliotecas anales de investigación, 11(11). 162. file:///C:/Users/doc-b1p8-

01/Downloads/Dialnet-

GestionDeInformacionYDelConocimien toParaLaTomaDeDe-

$5704545 \% 20(1)$.pdf

Suleman, L. (2006). Reflexiones sobre el concepto de conocimiento. http://www.knowledgeboard.com/\&sa= $\mathrm{X}$

$\& o i=$ translate $\&$ resnum $=1 \& \mathrm{ct}=$ result $\& \mathrm{pr}$ ev=/search\%3Fq\%3Dwww.knowledgeb oard.com/\%26hl\%3Des
Torres, K. y Lamenta, P. (2015). La gestión del conocimiento y los sistemas de información en las organizaciones. Negotium, 11(32), 3-20. https://www.redalyc.org/pdf/782/78246 590001.pdf

Torres, K., Lamenta, P. y Hamidian, B. (2018). Clima organizacional como gestión del conocimiento. Sapienza Organizacional, $\quad$ 5(9), 159-172. https://www.redalyc.org/articulo.oa? $\mathrm{id}=$ 5530/553056570008

Valero, J., López, M. y Pirela, G. (2017). Sistema de gestión de conocimiento para comunidades académicas. Opción, 33(82), $550-562$.

https://www.redalyc.org/articulo.oa?id= 310/31053180024

Yong, J., Rodríguez, F. y Ruso, A. (2017). El capital intelectual como factor de innovación y de impacto social en las universidades. Una mirada al Ecuador. Cuaderno Venezolano de Sociología, 26(4), 205-219.

https://www.semanticscholar.org/paper/ El-capital-intelectual-como-factor-deinnovaci\%C3\%B3n-y-Amaya-

$\underline{\text { Samaniego/ee4776c261b96520dd96fa4f }}$ 2d9ea559afc48f8a 